\title{
Aulas práticas e produções artísticas como ferramentas de conscientização ambiental em cursos de graduação
}

\section{Sidney Marcelo Victor de Andrade ${ }^{1}, *$, Victor Sacramento Dias $^{1}$, Gabriella Agnes de Oliveira Pester Gomes ${ }^{1}$, Danielle de Lima Viana ${ }^{1}$ e Paulo Guilherme Vasconcelos de Oliveira ${ }^{2}$}

${ }^{1}$ Universidade Federal Rural de Pernambuco. Departamento de Pesca e Aquicultura. Laboratório de Etologia de Peixes. Rua Dom Manuel de Medeiros, S/No. Dois Irmãos. Recife-PE, Brasil (CEP 52171-900). *E-mail: sidney_marcelo95@hotmail.com.

${ }^{2}$ Universidade Federal Rural de Pernambuco. Departamento de Pesca e Aquicultura. Rua Dom Manuel de Medeiros, S/№. Dois Irmãos. Recife-PE, Brasil (CEP 52171-900).

Resumo. Discussões acerca da conservação do meio ambiente têm sido frequentes nos últimos anos, consequentemente, os impactos ambientais gerados por resíduos sólidos estão sempre em pauta. As aulas práticas possuem extrema importância na conscientização ambiental dos discentes desde o ensino escolar até a graduação, incentivando a criatividade para minimizar os impactos supracitados. 0 presente artigo buscou desenvolver a conscientização ambiental de discentes de graduação em engenharia de pesca por meio de aulas práticas e produção artística. Os discentes foram separados em grupos e divididos em parcelas de $200 \mathrm{~m}$ cada. Em seguida, os alunos coletaram os resíduos presentes nas áreas, pesaram e separaram parte do material para produção de um quadro por meio de colagem. Foram coletados cerca de $21 \mathrm{~kg}$ de resíduos, sendo o vidro o mais pesado dentre eles, apesar disto, foi identificado que plásticos, tanto fragmentados quanto inteiros foram encontrados em maior quantidade. Nas telas as equipes buscaram representar diferentes ambientes marinhos e suas diversidades, criticaram a formação dos resíduos sólidos gerados pelo consumismo, os efeitos da pesca fantasma sobre espécies marinhas e os impactos antrópicos gerados pela atividade pesqueira. A pesquisa conseguiu alcançar o objetivo, incentivando a criatividade dos discentes em discutir assuntos importantes para o meio ambiente.

Palavras-chave: Resíduos sólidos; Pesca fantasma; Lixo marinho; Conservação; Oceano limpo.

Recebido $04 / 08 / 2020$

Aceito

$10 / 04 / 2021$

Disponível on line $12 / 04 / 2021$

Publicado $30 / 04 / 2021$

Acesso aberto

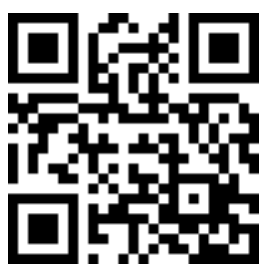

ORCID

D) 0000-0002-7335-1157 Sidney Marcelo Victor de Andrade

ISSN 2359-1412/RBGAS-2020-0118/2021/8/18/16/265

Rev. Bras. Gest. Amb. Sustent.

http://revista.ecogestaobrasil.net 
Abstract. Practical classes and artistic productions as tools for environmental awareness in undergraduate courses. Debates regarding environmental conservation have been frequent in the last years. Therefore, the impacts generated by solid waste are always on the agenda. Practical classes are essential in students' environmental awareness from childhood education to grad school, encouraging creativity to minimize impact. This work developed the perception of undergraduate students in fishing engineering through practical classes and artistic production. The students were separated into groups, responsible for an area of $200 \mathrm{~m}$ each. They then collected the residues found in the regions, weighed, and isolated part of the material to produce a collage painting. About $21 \mathrm{~kg}$ of waste were collected, with glass being the heavier among them, although it was identified that plastics, both fragmented and whole, were found in greater quantity. The teams tried to represent different marine environments and their diversity, criticized the formation of solid waste generated by consumerism, the effects of ghost fishing over marine species, and the anthropic impacts provoked by irresponsible fishing. The research managed to achieve its objective, encouraging its creativity in discussing important issues for the environment.

Keywords: Solid resides; Ghost fishing; Marine debris; Conservation; Clean ocean.
D 0000-0001-5952-8588

Victor Sacramento

Dias

(1) 0000-0002-0373-6254

Gabriella Agnes de

Oliveira Pester Gomes

D 0000-0003-2357-5280

Danielle de Lima Viana

(1) 0000-0001-7697-2111

Paulo Guilherme

Vasconcelos de

Oliveira

\section{Introdução}

0 tema relativo a conservação do meio ambiente tem sido fortemente discutido na sociedade nos últimos anos, o qual, dentro da educação em ciências e meio ambiente, é gerado a partir do conhecimento técnico científico (Reigota, 2007), todavia, as abordagens sobre o tema sobrescrito em muitas instituições de ensino não demonstram clareza e, por muitas vezes, não são apresentadas como indicado pelas políticas de ensino e aprendizagem, isto acontece porque o sistema de transversalidade para a melhoria de ensino não é conhecido por muitos dos educadores e, aqueles que o conhecem, poucos aplicam em parceria com as instituições de ensino (Bernardes e Prieto, 2010; Berrio, 2015; Fragoso e Nascimento, 2017).

As aulas de campo são consideradas como uma importante estratégia no ramo da educação desde o ensino básico ao superior, visto que motiva os discentes por meio do contato direto com a área de conhecimento de interesse (Viveiro e Diniz., 2009; Viveiro et al., 2009; Carvalho et al., 2016). A prática de campo das ciências ambientais, por sua vez, atua na motivação dos estudantes focados no interesse da conscientização ambiental, além de aguçar o olhar crítico para as interações ecossistêmicas, descobertas de espécies, atuação em unidades de conservação entre outros, permitindo proporcionar maiores oportunidades para a formação do conhecimento e compreensão do tema estudado (Miranda et al., 2010; Berrio, 2015).

Este olhar crítico normalmente é gerado a partir do tema de cada aula prática e do ambiente em que os discentes estão inseridos, os ecossistemas costeiros, por exemplo, afetados excessivamente pelo aumento em 49\% dos resíduos sólidos no Brasil (plástico, isopor, papel, vidro, madeira e outros), muito por causa do aumento populacional e um sistema equivocado de produção, confecção de produtos artificiais e sintéticos, estacagem 
e descarte desses resíduos, sem a preocupação do possível impacto ambiental (Coe e Rogers, 2000; Amorim, 2010; Waldman, 2010; Di Creddo, 2012).

Tornar a comunidade acadêmica ciente dos problemas gerados pela própria sociedade é um dos focos das aulas práticas realizadas dentro das Áreas de Ciências Agrárias e Biológicas, mais especificamente no Curso de Engenharia de Pesca, da Universidade Federal Rural de Pernambuco, que é alvo do presente estudo. Apesar disso, os resultados da conscientização ambiental promovida pela instituição pouco são conhecidos pela sociedade. Desta forma, o presente artigo teve como objetivo abordar uma nova vertente da educação ambiental a partir de dados gerados nas aulas práticas, que, além do conceito científico, o conceito artístico foi utilizado para destinar parte dos resíduos encontrados, bem como expressar opiniões e ideias sobre a poluição marinha.

\section{Material e métodos}

Foi realizada uma aula prática na Paraia de Maracaipe, Município de Ipojuca, situado no litoral sul do Estado de Pernambuco, Brasil, em dois dias (13 e 14 de julho de 2019), com os alunos do sexto período do Curso de Engenharia de Pesca, da Universidade Federal Rural de Pernambuco (UFRPE), referentes às disciplinas de Ecossistemas Costeiros e Oceanografia Abiótica, com temas de formações praiais, ecossistêmicas e sensibilização ambiental. Ao todo, 16 discentes participaram participaram da aula prática e foram separados em quatro grupos para realização das coletas de resíduos sólidos no supralitoral da Praia de Maracaípe (Figura 1).

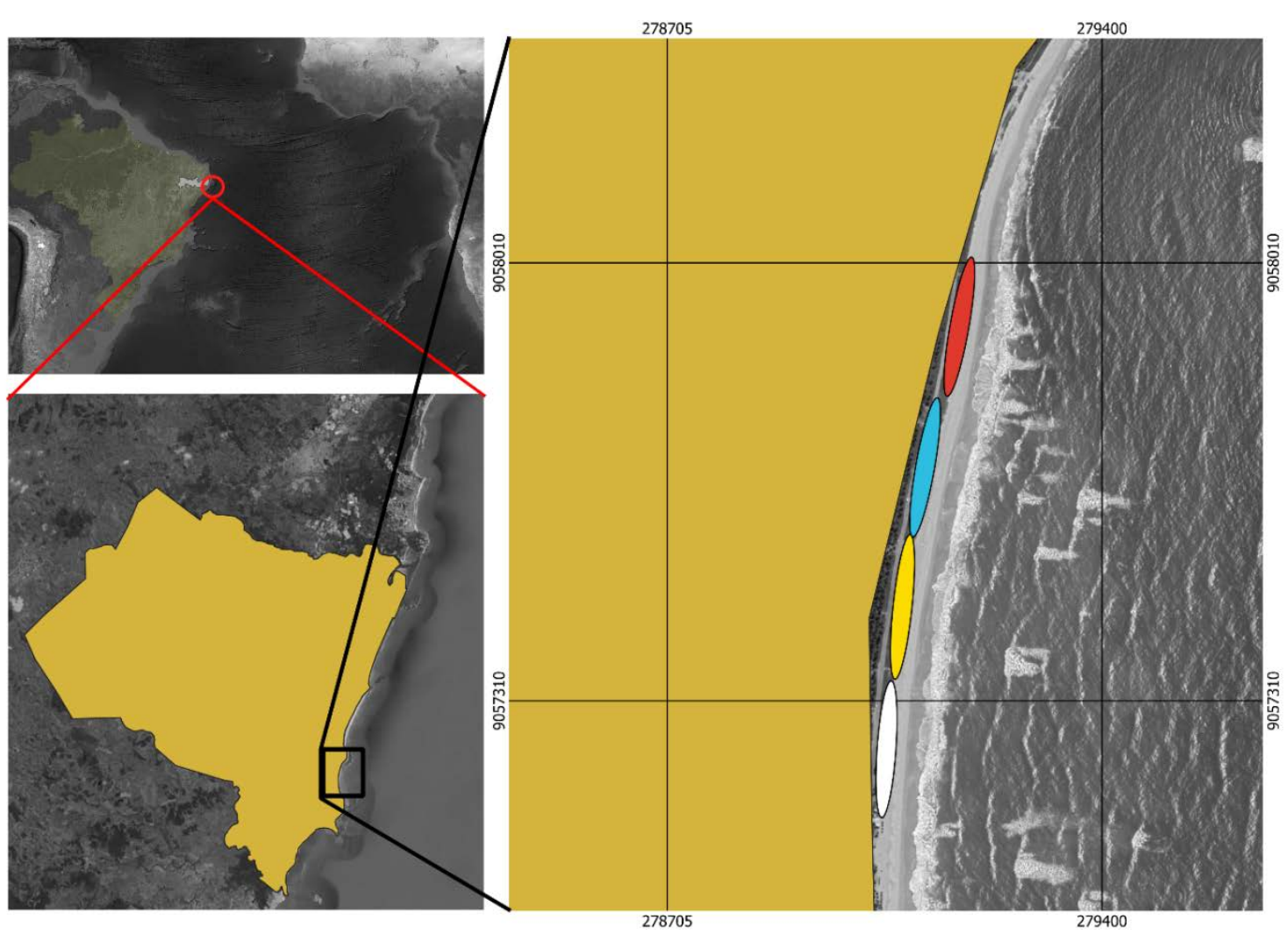

Figura 1. Área de coleta de resíduos sólidos, Praia de Maracaípe, Ipojuca-PE. Fonte: Google Earth. 
Foi coberta uma área de aproximadamente $2 \mathrm{~km}^{2}$, dividida em quatro parcelas de $200 \mathrm{~m}$ x $10 \mathrm{~m}$, no qual, cada grupo ficou responsável por realizar buscas intensivas em cada região, coletando todos os resíduos sólidos encontrados. 0 material foi classificado em plástico, esponja, látex, tecido, orgânico, alumínio, papel, vidro, isopor, madeira, metal, pedra, resíduos de pesca e outros (materiais não identificados em consequência do alto nível de degradação) e pesados em seguida. Não foi possível obter a frequência absoluta e relativa dos dados devido ao alto grau de fragmentação.

Com fim avaliativo, os grupos foram encorajados a aproveitar parte dos resíduos coletados na construção de um quadro por método de colagem, com a intenção de levar ao público geral, aspectos gráficos que refletissem em alguma vertente da conscientização ambiental, seja ela conservação, poluição, administração ambiental e outros.

\section{Resultados}

\section{Distribuição e volume de resíduos sólidos}

Cerca de $21 \mathrm{~kg}$ de resíduos foram coletados durante as atividades, com destaque para o vidro $(5,13 \mathrm{~kg})$, látex $(4,88 \mathrm{~kg})$ e plástico $(3,44 \mathrm{~kg})$, que representaram mais de $50 \%$ do total coletado (Figura 2).

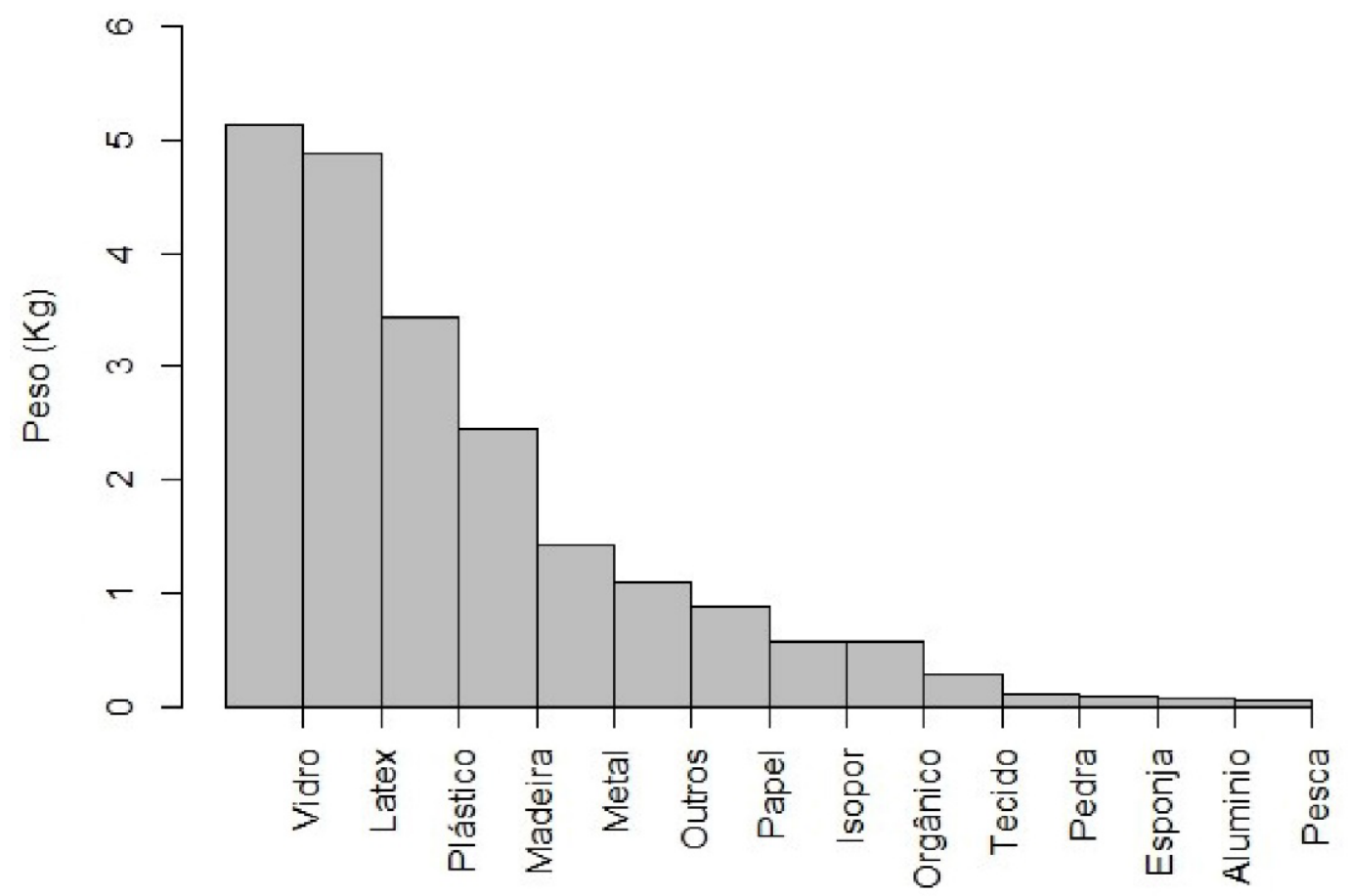

Figura 2. Volume total de de resíduos sólidos $(\mathrm{kg})$ coletados, por grupos, nas áreas de coletas da Praia de Maracaípe, Ipojuca-PE.

Apesar da região de estudo estar muito próxima de bares e restaurantes, o que poderia influenciar na distribuição do lixo no supralitoral, não foi observado um padrão de variação de peso entre as parcelas, sendo encontrado um alto volume de resíduos em todas as delimitações da praia (Figura 3). Esse acúmulo pode estar ligado à utilização de toda a extensão de praia para o lazer. Além disso, não foram observadas lixeiras ou outros pontos de descarte apropriados no local, o que pode elevar a taxa de poluição antrópica nas praias. 


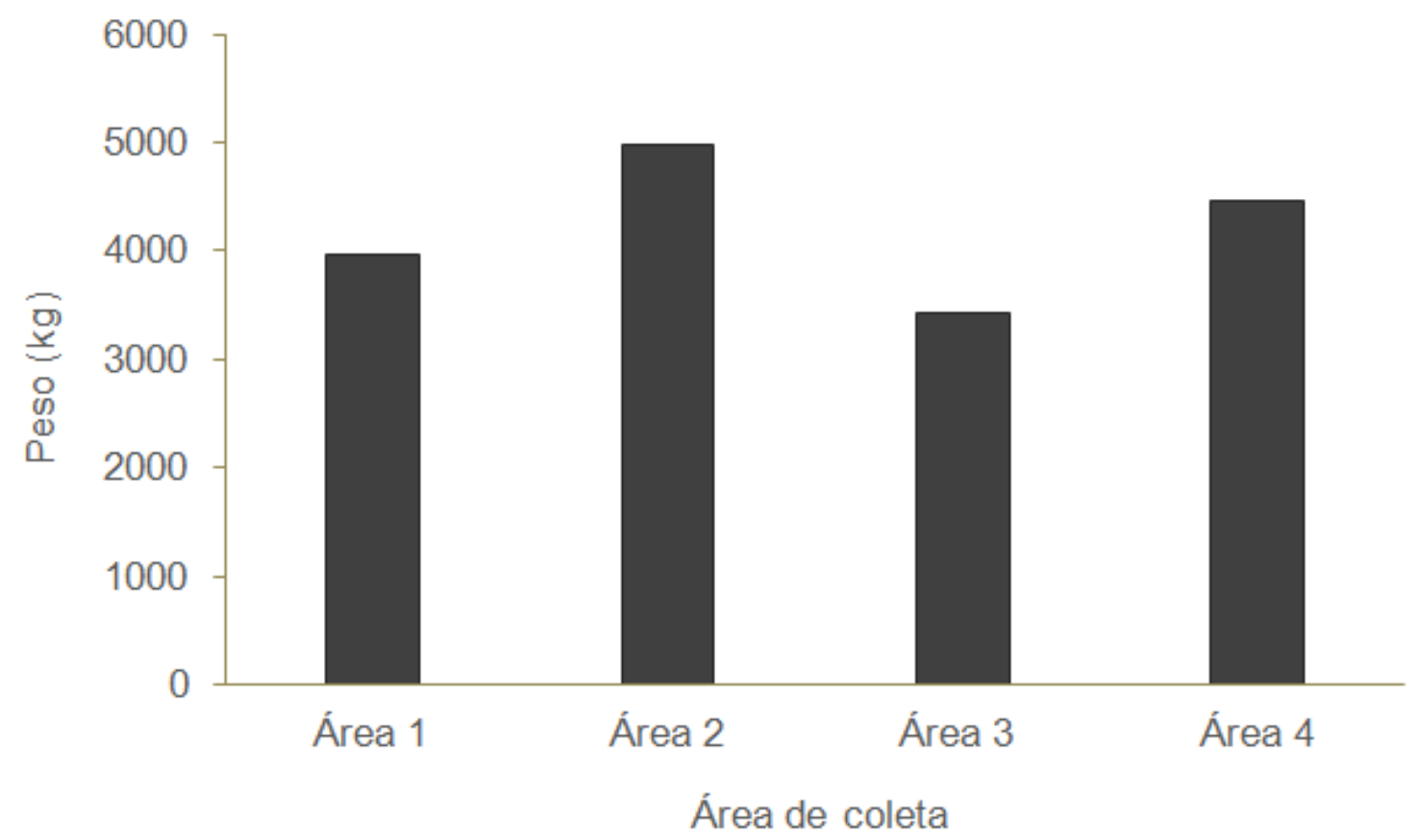

Figura 3. Peso (kg) dos resíduos sólidos coletados, por área de coleta, na Praia de Maracaípe, Ipojuca-PE.

\section{Construção de obras de arte com finalidade educacional}

Foram confeccionados quatro quadros com colagens dos resíduos sólidos coletados, cada um buscando contextualizar temas distintos da poluição dos ecossistemas costeiros (Figura 4). 0 primeiro grupo buscou representar diferentes ambientes marinhos e suas diversidades, com representação dos ambientes bentônicos e pelágicos, bem como sua fauna e flora. Um maior destaque foi dado para a relação clássica dos quelônios e os resíduos plásticos, já que esses animais tendem a confundir sacolas plásticas com cnidários pelágicos.

O segundo grupo confeccionou uma arte sob o argumento dos efeitos do consumismo, intitulada "O Preço do Lixo.". Atentando para os mais diversos resíduos, contendo desde lixo hospitalar a materiais de couro (exemplo: carteira), a analogia foi realizada no preço pago pelos ambientes naturais em razão do consumismo desenfreado da população.

0 terceiro grupo buscou representar os efeitos da pesca sob um recorte da fauna que não é alvo da atividade pesqueira. A representação da tartaruga emaranhada com artes de pesca evidencia o pensamento crítico dos alunos quanto aos efeitos da pesca acidental e da pesca fantasma. Os aparelhos de pesca perdidos, abandonados ou descartados (APPAD) impactam diretamente os ciclos de vida em diversos ambientes, desde a superfície até regiões profundas, causando ferimentos graves e mortalidade.

0 quarto grupo abordou a questão da pesca e a modificação dos ambientes. A representação da pesca de arrasto e seus efeitos sob os ecossistemas bentônicos refletem a sensibilidade dos alunos em relação aos impactos gerados por esse tipo específico de pescaria. A contextualização dos efeitos pelo uso dos equipamentos elucida tanto o grau de envolvimento dos alunos com a atividade quanto a compreensão da ideia proposta. 

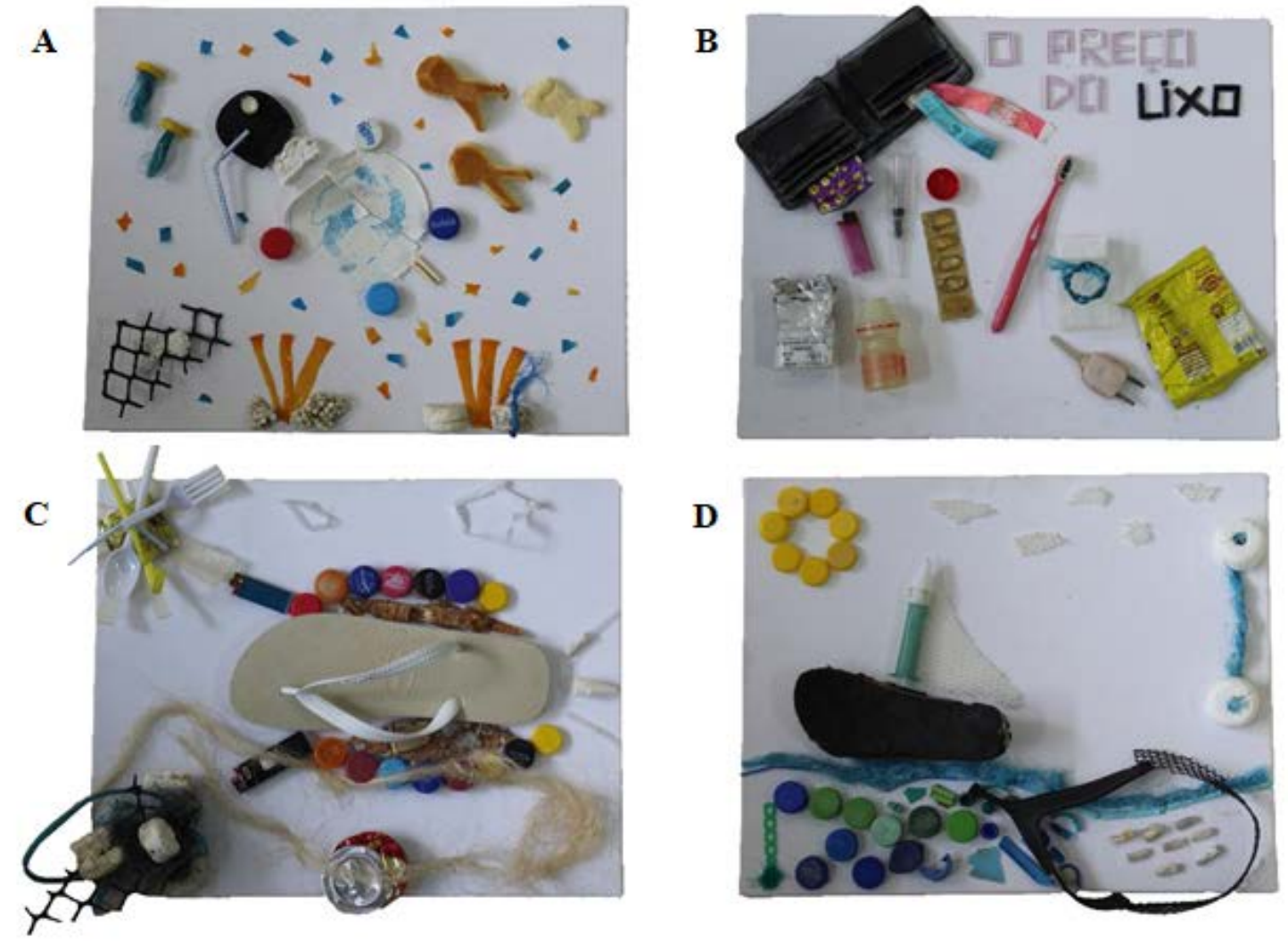

Figura 4. Quadros realizados a partir dos residuos encontrados pelos discentes. A - 1o grupo; B - 2oㅡ

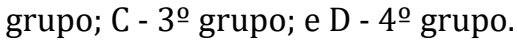

\section{Discussão}

Santana Neto et al. (2001), em trabalho realizado na Ilha de Itaparica-BA, região turística, assim como a avaliada no presente estudo, encontrou o resíduo plástico em maior quantidade. Baptista Neto e Fonseca (2011) observaram o mesmo padrão para Baia de Guanabara, Rio de Janeiro, onde o plástico representava cerca de $70 \%$ do total coletado nas praias. Os resultados do presente trabalho acompanharam a tendência mundial, onde o plástico é o resíduo mais encontrado em praias (Santana Neto et al., 2001), em que pese, este não tenha sido o resíduo com maior volume. Este fato pode ter acontecido devido ao interesse dos alunos em priorizar a coleta de resíduos de maiores volumes e/ou pesos, produtos em vidro.

Para Nunes et al. (2018) e Morin et al. (2007) a arte é a expressão da inteligência e sensibilidade proporcionando ao ser humano uma maior capacidade sencitiva e cognitiva por meio da liberdade de criação. Indicando que os alunos participantes desta atividade de campo e das ideias criativas da atividade artística, foram induzidos a um maior senso crítico e cognitivo.

De acordo com André (2011) uma arte contestadora pode desenvolver um senso crítico diferente e, consequentemente, novas respostas para questões consideradas normais pela sociedade e/ou já respondidas. A atividade realizada pelos alunos do Curso de Engenharia de Pesca trata-se de uma arte contestadora com referência na importância da conservação ambiental dos ecossistemas costeiros, combatendo a normalização do descarte de resíduos nas praias agravado pelo aumento do consumo, turismo e aparelhos de pesca perdidos, abandonados ou descatados (APPAD). 


\section{Conclusão}

A contextualização in loco, proposta pela atividade prática, contribuiu para uma melhor absorção dos conceitos expostos nesta atividade. A adição da atividade artística impulsionou o senso crítico dos alunos participantes, tornando o exercício multidisciplinar e capaz de versar por temas com menor abordagem nas disciplinas da grade curricular do curso, como os efeitos sociais da degradação da paisagem através da poluição. Conclui-se que a percepção dos alunos, acerca dos efeitos dos resíduos sólidos descartados em ambientes costeiros, atendeu as expectativas dos autores, vide os resultados obtidos tanto nas atividades artísticas, em que os alunos propuseram não só representações gráficas, mas também os conceitos expostos durante as aulas teóricas das disciplinas envolvidas na atividade de campo, como na coleta de resíduos, onde os alunos buscaram representar os mais diversos grupos.

\section{Conflito de interesses}

Os autores declaram não haver confito de interesses.

\section{Referências}

Amorim, A. P.; Albuquerque, B. M.; Gautério, D. T.; Jardim, D. B.; Morrone, E. C.; Souza, R. M. Lixão municipal: abordagem de uma problemática ambiental na Cidade do Rio Grande-RS. Ambiente \& Educação, v. 15, n. 1, p. 159-178, 2010.

André, C. Arte, biopolítica e resistência. Revista Brasileira de Estudos da Presença, v. 1, n. 2, p. 426-442, 2011. https://doi.org/10.1590/2237-266021497

Baptista Neto, J. A.; Fonseca, E. M. Variação sazonal, espacial e composicional de lixo ao longo das praias da margem oriental da Baía de Guanabara (Rio de Janeiro) no período de 1999-2008. Revista de Gestão Costeira Integrada, v. 11, n. 1, p. 31-39, 2011. https://doi.org/10.5894/rgci189

Bernardes, M. B. J.; Prieto, E. C. Educação ambiental: disciplina versus tema transversal. Revista Eletrônica do Mestrado em Educação Ambiental, v. 24, p. 173-185, 2010.

Berrio, A. S. Construcción del conocimiento científico a partir del discurso de aula en la enseñanza de las Ciencias Naturales. Revista Palabra, v. 4, n. 1, p. 80-91, 2015.

Carvalho, M. E. A.; Franco, M. R.; Zanatta, S.; Oliveira, R. A.; Pipitone, M. A. P. O rio e a escola: uma experiência de extensão universitária e de educação ambiental. Química e Sociedade, v. 39, n. 2, p. 112-119, 2017. https://doi.org/10.21577/0104-8899.20160067

Coe, J. M.; Rogers, D. B. Marine debris: Sources, impacts and solutions. New York: Springer, 2000.

Di Creddo, E. Lixo urbano: um desafio ambiental. São Leopoldo: IHU On-Line, 2012. Disponível em: <http://www.ihu.unisinos.br/entrevistas/508034-lixo-urbano-umdesafio-ambiental-entrevista-especial-com-eleusis-dicreddo>. Acesso em: 30 mar. 2020.

Fragoso, E.; Nascimento, E. C. M. Educação ambiental no ensino e na prática escolar da Escola Estadual Cândido Mariano - Aquidauana/MS. Revista Saúde e Meio Ambiente, v. 5, n. 2, p. 105-126, 2017. https://doi.org/10.14295/ambeduc.v23i1.6988

Miranda, F. H. F.; Miranda, J. A.; Ravaglia, R. Abordagem interdisciplinar em educação ambiental. Revista Práxis, v. 2, n. 4, p. 11-16, 2017. https://doi.org/10.25119/praxis-2-4922 
Morin, A.; Gadoua, G.; Potvin, G. Saber, Ciência e ação. São Paulo: Cortez, 2007.

Nunes, L.S. R.; Bomfim, A. M.; Fonseca, G. R.S. Educação ambiental crítica e arte participativa: a construção de reflexão e ação em uma escola da Baixada Fluminense. Amazônia - Revista de Educação em Ciências e Matemáticas, v. 14, n. 30, p. 42-58, 2018. https://doi.org/10.18542/amazrecm.v14i30.5921

Reigota, M. 0 estado da arte da pesquisa em educação ambiental no Brasil. Revista

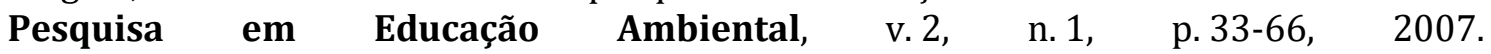
https://doi.org/10.18675/2177-580X.vol2.n1.p33-66

Santana Neto, S. P.; Silva, I. R.; Livramento, F. C. Padrões de deposição e origens do lixo marinho em praias da Ilha de Itaparica-Bahia. Anais do V simpósio Brasileiro de Oceanografia, 2011.

Viveiro, A. A.; Diniz, S. As atividades de campo no ensino de Ciências: reflexões a partir das perspectivas de um grupo de professores. In: Nardi, R. (Org.). Ensino de Ciências e Matemática, I: temas sobre a formação de professores. São Paulo: Editora UNESP; São Paulo: Cultura Acadêmica, 2009. p. 27-42. Disponível em: <http://books.scielo.org/id/g5q2h/pdf/nardi-9788579830044-03.pdf>. Acesso em: 14 abr. 2020.

Viveiro, A. A.; Eugênio, R.; Diniz, S. Atividades de campo no ensino das ciências e na educação ambiental: refletindo sobre as potencialidades desta estratégia na prática escolar. Ciência em Tela, v. 2, n. 1, p.1-12, 2009. Disponível em: <http://www.cienciaemtela.nutes.ufrj.br/artigos/0109viveiro.pdf>. Acesso em: 14 abr. 2020.

Waldman, M. Lixo: cenários e desafios. São Paulo: Cortez, 2010.

(CC) Informação da Licença: Este é um artigo Open Access distribuído sob os termos da Licença Creative Commons Attribution, que permite uso irrestrito, distribuição e reprodução em qualquer meio, desde que a obra original seja devidamente citada. 